\title{
Influencia de la calidad de los agregados y tipo de cemento en la resistencia a la compresión del hormigón dosificado al volumen
}

\author{
Influence of aggregate quality and type of cement in compressive strength of concrete \\ proportioned by volume
}

\author{
Jorge L. Santamaría ${ }^{(0)}$, Byron Adame ${ }^{(i)}$, César Bermeo \\ ${ }^{1}$ Facultad de Ingeniería y Ciencias Aplicadas, Universidad Central del Ecuador, Quito, Ecuador, 170521; bsadame@uce.edu.ec; \\ cabermeo@uce.edu.ec
}

*Correspondencia: jsantamaria@uce.edu.ec

\begin{abstract}
Citación: Santamaría, J., Adame, B. \& Bermeo, C., (2021). Influencia de la calidad de los agregados y tipo de cemento en la resistencia a la compresión del hormigón dosificado al volumen. Novasinergia. $\quad 4(1)$. 91-101. https://doi.org/10.37135/ns.01.07.05
\end{abstract}

Recibido: 13 abril 2021

Aceptado: 20 mayo 2021

Publicado: 01 junio 2021

Novasinergia

ISSN: 2631-2654

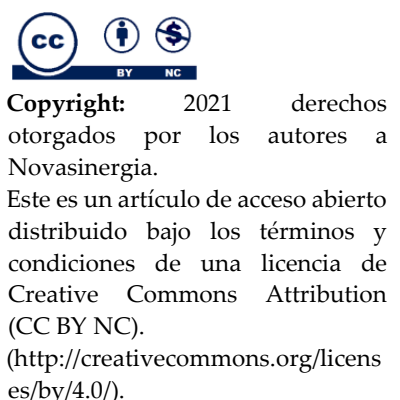

Resumen: El hormigón es el material más utilizado en construcción y su calidad debe asegurarse cuando se fabrique en obra. La Guía de Diseño 7 recomienda dosificaciones al volumen a los llamados no profesionales de la construcción para la fabricación de hormigón estructural in situ. El propósito es promover buenas prácticas en la elaboración del hormigón, debido a un alto índice de construcciones informales existentes en el Ecuador, de cumplir con la normativa vigente. El objetivo principal del estudio es demostrar que la calidad del hormigón puede verse seriamente afectada si se utiliza únicamente dosificación al volumen, sin considerar, las propiedades de sus componentes. La teoría de diseño de experimentos (DOE) fue empleada para fabricar mezclas con una misma dosificación al volumen, pero utilizando diferentes propiedades de agregados y tipos de cemento de uso general disponibles en el mercado. Un modelo matemático resultante del análisis del diseño factorial permitió determinar el impacto de los referidos factores en la resistencia a la compresión del hormigón $\left(f^{\prime} c\right)$, encontrándose una afectación significativa en un rango de $-13.7 \%$ a $28.4 \%$. Los profesionales y no profesionales de la construcción deben considerar dicho impacto y establecer una dosificación propia para cada proyecto.

Palabras clave: Áridos para el hormigón, diseño de experimentos, dosificación de mezclas por volumen, resistencia del hormigón a compresión.

\begin{abstract}
Concrete is the most widely used material in construction. Its quality must be assured when it is manufactured on-site. Design Guide 7 recommends batching to volume for non-professional construction workers to manufacture structural concrete in situ. This study aims to promote good practices in the manufacture of concrete due to the high rate of informal construction in Ecuador and to comply with current regulations. The study's main objective is to demonstrate that the quality of concrete can be seriously affected if only batching by volume is used, without considering the properties of its components. The design of experiments (DOE) theory was employed to produce mixes with the same volume proportioning but using different aggregate properties and types of cement commonly available in the market. A mathematical model resulting from the factorial design analysis allowed determining the impact of the referred factors on the concrete compressive strength $\left(f^{\prime} \mathrm{c}\right)$, finding a significant affectation in a range of $-13.7 \%$ to $28.4 \%$. Construction professionals and nonprofessionals should consider this impact and establish their dosage for each project.
\end{abstract}

Keywords: Aggregates for concrete, concrete compressive strength, concrete, design of experiments, volume mixture proportioning. 


\section{Introducción}

En la actualidad, el hormigón es el material más utilizado en la industria de la construcción y su fabricación debe ser cuidadosa a fin de obtener la calidad deseada. El esfuerzo de compresión $\left(f^{\prime} c\right)$ es generalmente el parámetro de calidad a ser considerado por ingenieros civiles en la ejecución de proyectos de infraestructura física debido a su facilidad para ser medido. La calidad del hormigón no depende solamente en la dosificación utilizada (cantidad de agua, cemento y agregados) y en las propiedades de sus componentes; sino también, en los procesos de colocación, compactación y curado (Mehta \& Monteiro, 2006).

El hormigón se lo prepara usualmente utilizando una dosificación al peso o al volumen, dependiendo si es premezclado o fabricado in situ. El Instituto Americano del Concreto (ACI por sus siglas en inglés) recomienda que el hormigón no se fabrique en obra; sino, en una planta especializada para el efecto (hormigón premezclado) por motivos de control de calidad. Sin embargo, en la práctica, casi siempre se fabricará hormigón en obra. En el Ecuador el porcentaje de construcciones informales es alto, especialmente en la capital de la república donde existe el 70\% de construcciones informales; de las cuales el 91.8\% están fabricadas de hormigón armado (Carvajal, 2019). En este caso, se debe considerar que una construcción es informal cuando no cuenta con el respectivo permiso de construcción emitido por los respectivos cabildos (Municipio de Quito, 2019).

Se recomienda que la fabricación del hormigón se la realice al peso y no al volumen. Según Kosmatka, Kerkhoff \& Panarese (2011) existen variaciones en el contenido de humedad, peso específico y capacidad de absorción, por lo que el peso aparente suelto y compactado de los agregados pueden variar el volumen aparente que ocupan los agregados al momento de fabricar hormigón. Otra razón para dosificar el hormigón al peso es que la calidad de los agregados pétreos utilizados usualmente no está garantizada. Como la mayoría de los agregados se entregan la obra en condiciones húmedas, pueden ocurrir grandes variaciones en las cantidades de la mezcla para el hormigón si se dosifica al volumen. Por esta razón se recomienda la dosificación al peso y se realicen las correcciones por humedad. De lo indicado se desprende que se debe tener muy en cuenta la variabilidad de calidad del hormigón cuando se lo fabrique utilizando dosificación al volumen, ya que esta es la principal fuente de variabilidad del hormigón (De Larrard, 2011).

La Secretaría de Gestión de Riesgos (SGR), el Ministerio de Desarrollo Urbano y Vivienda (MIDUVI), el Programa de las Naciones Unidas para el Desarrollo (PNUD) y la Oficina de Ayuda Humanitaria y Protección Civil de la Comisión Europea $(\mathrm{ECHO})$ publicaron un conjunto de siete guías prácticas de implementación de la Norma Ecuatoriana de la Construcción (Ministerio de Desarrollo Urbano y Vivienda \& Secretaria de Gestión de Riesgos, 2016) con la finalidad de que los profesionales y no profesionales de la construcción del país puedan aplicar la NEC-15 (Ministerio de Desarrollo Urbano y Vivienda \& Cámara de la Industria de la Construcción, 2015) de manera adecuada. Debe entenderse que los profesionales de la construcción, ingenieros o arquitectos, son aquellos que recibieron formación especializada en el campo de la construcción, sin embargo, los llamados no profesionales de la construcción, maestros de obra u albañiles, son aquellos trabajadores que no han recibido capacitación formal en este campo. Todos sus conocimientos han sido mayormente adquiridos por experiencia. Estos no profesionales de la construcción son aquellos encargados usualmente de edificar las llamadas construcciones informales sin contar con la supervisión de profesionales de la construcción ni los respectivos permisos de construcción.

Debido al gran porcentaje de edificaciones construidas de manera informal, la Guía de Diseño 7 (Ministerio de Desarrollo Urbano y Vivienda \& Secretaria de Gestión de Riesgos, 2016) pretende brindar conocimiento necesario a los no profesionales de la construcción con el propósito de incentivar prácticas de construcción idóneas tendientes a cumplir con la normativa vigente 
establecida por la NEC-15 (Ministerio de Desarrollo Urbano y Vivienda \& Cámara de la Industria de la Construcción, 2015). En este marco y en relación a la fabricación de hormigón, la referida guía recomienda que para la fabricación de hormigón a ser utilizado en elementos estructurales se utilice una "receta" o dosificación preestablecida. Dicha dosificación se da "al volumen" a través de la utilización de unidades volumétricas llamadas parihuelas. Las mencionadas parihuelas son paralelepípedos rectangulares de aristas iguales cuyas dimensiones son usualmente de $30 \mathrm{~cm}$ por lado. Se la concibió de esta manera en razón de que en una parihuela contiene un saco de cemento de $50 \mathrm{~kg}$.

La dosificación sugerida en la Guía de Diseño 7 (Ministerio de Desarrollo Urbano y Vivienda \& Secretaria de Gestión de Riesgos, 2016) brinda varias dosificaciones al volumen dependiendo del tipo de elemento estructural a ser construido. Por ejemplo, para la fabricación de hormigón para losas, vigas y columnas, la dosificación es de 1 saco de 50 kilogramos de cemento, 25 litros de agua potable, 2 parihuelas de arena al ras y 2.5 parihuelas de ripio al ras, sin embargo, no se hace referencia a la resistencia esperada a la compresión $\left(f^{\prime} c\right)$ ni a las condiciones de humedad en que deben estar los agregados. Cuando se fabrica hormigón en obra se debe considerar las propiedades de los materiales constituyentes del hormigón; es decir, se debe disponer de la caracterización del agregado fino y agregado grueso, tipo de cemento, y aditivos con la finalidad de realizar un diseño de mezclas que cumpla con las especificaciones técnicas requeridas en los planos de diseño.

A través del tiempo se han desarrollado varios métodos para realizar diseño de mezclas de hormigón, algunos son netamente empíricos, mientras que otros son proporcionados por reconocidas organizaciones o códigos como por ejemplo el Comité 211 del American Concrete Institute (ACI, 2009), lo cual implica que no existe una "receta general" para fabricar hormigón. Se debe establecer las proporciones de los componentes del hormigón basados exclusivamente en las propiedades de los materiales disponibles; entre las que se puede mencionar los módulos de finura, resistencia al desgaste (abrasión), peso específico y capacidad de absorción (porosidad), y densidades aparentes de los agregados. El resultado de una dosificación de mezclas de hormigón determina las cantidades de materiales por metro cúbico; es decir, una dosificación al peso, a partir de la cual de debe realizar las correcciones por humedad pertinentes y poder llegar a establecer una dosificación al volumen.

El objetivo principal del presente estudio es cuantificar la variabilidad de la resistencia del hormigón $\left(f^{\prime} c\right)$ utilizando una misma dosificación al volumen propuesta en la Guía de Diseño 7 (Ministerio de Desarrollo Urbano y Vivienda \& Secretaria de Gestión de Riesgos, 2016) para losas, vigas y columnas a través de la utilización de la teoría de DOE. Un modelo matemático resultante de un diseño factorial completo será utilizado para cuantificar el impacto de las propiedades de los agregados pétreos y tipo de cemento en la resistencia a la compresión del hormigón.

\section{Metodología}

La figura 1 ilustra la metodología que se desarrolló a fin de alcanzar el objetivo propuesto. Primeramente, se parte de una dosificación al volumen constante sugerida por la Guía de Diseño 7 (Ministerio de Desarrollo Urbano y Vivienda \& Secretaria de Gestión de Riesgos, 2016) según se mencionó anteriormente. Se establecen los factores que se van estudiar a través de un diseño factorial completo $2^{k}$ que permitirá elaborar un modelo matemático para estimar la resistencia a la compresión del hormigón $\left(f^{\prime} c\right)$ y poder evaluar el impacto en la resistencia a la compresión que tienen sus componentes cuando se trabaja con una dosificación al volumen prestablecida. 

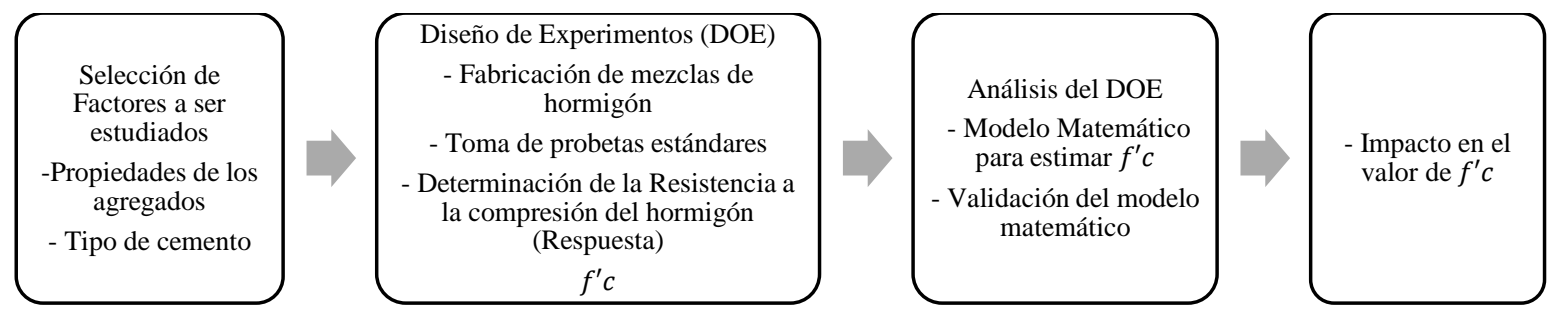

Figura 1: Metodología de estudio

\subsection{Selección de Factores a ser estudiados}

Para estudiar la influencia de los agregados pétreos y del tipo de cemento en la calidad del hormigón se han escogido los siguientes factores, considerando la granulometría y dureza de los agregados representada por el módulo de finura y el \% de desgaste del agregado grueso conocido como abrasión; así como también, dos tipos de cementos para uso general disponibles en el mercado local. Para la selección de los agregados a ser utilizados en este estudio se consideró las canteras más utilizadas en el Distrito Metropolitano de Quito, como son, las canteras de la zona de San Antonio de Pichincha, Pifo y Guayllabamba. De las referidas canteras se procedió a realizar los respectivos ensayos de laboratorio y utilizar agregados con parámetros extremos de módulos de finura y porcentajes de desgaste. Dichos valores corresponden al promedio de tres ensayos realizados con el fin de validar los resultados. El contenido de humedad de los agregados fue "seco al aire" y se realizaron las correcciones respectivas de humedad al momento de la fabricación del hormigón a fin de colocar los agregados en condición "saturada superficie seca". De igual manera, los dos tipos de cemento seleccionados para la investigación corresponden a los fabricados por dos empresas productoras de cemento diferentes y que son los de mayor uso para la fabricación de hormigón estructural normal en el país.

1.- Módulo de Finura del Agregado Grueso (MFAG): Esta variable se la determinó utilizando la normativa ASTM C 136 (2019) con un nivel alto de $7.3(+1)$ y un nivel bajo de $6.6(-1)$.

2.- Módulo de Finura del Agregado Fino (MFAF): Esta variable se la determinó utilizando la normativa ASTM C 136 (2019) con un nivel alto de $3.4(+1)$ y un nivel bajo de $2.1(-1)$.

3.- Abrasión o Porcentaje al Desgaste ( $A B)$ : Esta variable se la determinó utilizando la normativa ASTM C 131 (2006) con un nivel alto de $50.8(+1)$ y un nivel bajo de $15.6(-1)$.

4. -Tipo de Cemento (TC): Dos tipos de cemento mayormente utilizados en la fabricación de hormigón para uso general fueron considerados en esta investigación. Cemento tipo GU (+1) y el cemento tipo IP (-1) elaborado en concordancia con la normativa NTE INEN 2380 basada en la norma ASTM (2020) y la NTE INEN 490 basada en la norma ASTM (2019c). Las especificaciones técnicas se las puede encontrar en Holcim (2020) y Unacem (2020).

\subsection{Diseño de Experimentos (DOE)}

Existen varias técnicas de experimentación que son llevadas a cabo por investigadores para alcanzar sus objetivos. Según Montgomery (2017), una de las prácticas más utilizadas para investigar la influencia de ciertos factores que afectan un determinado proceso es aplicando el conocimiento y la experiencia ya adquirida por el investigador aplicando el "mejor criterio "y otra donde los científicos hacer "variar un factor a la vez", manteniendo los otros constantes. Esto procedimientos no son eficientes ya que no identifican interacciones entre factores y requiere de un gran número de experimentos. Una interacción se produce cuando un factor no afecta en la misma intensidad un sistema en presencia de otro factor a diferente intensidad. La teoría de DOE es una técnica idónea de investigación a ser utilizada para investigar la influencia de factores que afectan o influyen la 
respuesta o salida de un sistema. A diferencia de otras técnicas, DOE permite al investigador no solamente identificar cuáles son los factores que afectan un sistema significativamente; sino también, encontrar posibles interacciones entre dos o más factores mediante la ejecución del menor número de pruebas, experimentos o ensayos.

Cuando se tiene varios factores que influencian un proceso se debe ejecutar un experimento denominado factorial. Según Cornell (2011), un diseño factorial estudia la variación producida en la respuesta cuando dos o más factores actúan simultáneamente a varios niveles, siendo su objetivo el medir los cambios en la respuesta para diferentes niveles de intensidad de los factores. Puede variar únicamente el nivel de un factor a la vez y mantener los otros constantes o a su vez pueden varias dos o tres factores simultáneamente. Los cambios en la respuesta producidos por cada factor son conocidos como efectos principales mientras que los cambios en la respuesta causados por interacción entre factores son llamados efectos de interacción.

Diseño $2^{k}$ es un diseño factorial completo donde el exponente $k$ es el número de factores a ser estudiados y la base 2 representa los niveles a los cuales los factores van a actuar. En este caso se estudian el efecto de todas las posibles combinaciones de los factores seleccionados tomando en consideración un nivel alto (+1) y un nivel bajo (-1). Debe entenderse como un diseño de experimentos a todo el conjunto de combinaciones o ensayos que se realizan. El número total de pruebas o ensayos a realizarse es 2 elevado al número de factores. Por ejemplo, si se tienen 5 factores a estudiarse, se necesitan $2^{5}$ experimentos, es decir 32 ensayos para llevar a cabo un diseño factorial completo. Se asume que la respuesta del experimento varia de forma lineal cuando un factor varía de nivel alto al bajo; es decir, la respuesta es una función lineal dentro del rango +1 al -1. Cada prueba o experimento a realizarse está conformado por una combinación única de factores actuando en nivel +1 o -1 , permitiendo cuantificar estadísticamente efectos principales o efectos causados por interacciones entre factores; así como también, la elaboración de modelos matemáticos que permitan estimar la resultante del sistema.

En diseños factoriales completos $\left(2^{k}\right)$ es importante examinar la magnitud y dirección de los efectos calculados a fin de establecer si los factores en estudio son importantes o no; en otras palabras, si son estadísticamente significativos o no. Esto se logra utilizando la técnica estadística del Análisis de Varianza (ANOVA), a pesar de que se puede utilizar las pruebas $t$ estudiante (Montgomery, 2017). Es muy recomendado el uso de software especializado para el diseño y análisis de experimentos factoriales, como, por ejemplo, Minitab 19 (Minitab, State College, PA: USA), mismo que fue manejado en la presente investigación.

Una vez calculados los efectos y realizados el ANOVA respectivo se puede discernir los efectos que son significativos a través del valor p estadístico, mismo que representa una probabilidad de que se descarte la hipótesis nula (Ho: El valor del coeficiente es igual a cero) de manera incorrecta. Es decir, si el valor de $p$ es menor que 0.05 en el cuadro del ANOVA, de descarta la hipótesis nula y se mantiene la hipótesis alternativa, implicando que el termino en referencia es estadísticamente significativo. A partir de esto se elabora un modelo matemático para un $2^{4}$ según la ecuación (1), asegurándose que todos los términos que contenga sean significativos.

$$
y=\beta_{o}+\beta_{1} x_{1}+\cdots+\beta_{4} x_{4}+\beta_{12} x_{1} x_{2}+\cdots+\beta_{34} x_{3} x_{4}+\beta_{123} x_{1} x_{2} x_{3}+\cdots+\beta_{234} x_{2} x_{3} x_{4}+\beta_{1234} x_{1} x_{2} x_{3} x_{4}
$$

Donde $y$ representa la respuesta, $\beta_{t s}$ son los efectos. Una vez que se tiene el modelo refinado, se debe realizar el chequeo de los residuales correspondientes a normalidad, independencia e igualdad de varianzas. ANOVA es usualmente robusto para normalidad, la independencia se la asegura a través de la randomización y la igualdad de varianza se las debe verificar a través de pruebas estadísticas. 
La tabla 1 contiene el diseño factorial completo para cuatro factores $2^{4}$ donde el nivel alto y bajo están codificados por +1 y -1 respectivamente. Los 16 experimentos (Un experimento es una mezcla) de hormigón fueron realizados en el Laboratorio de Ensayo de Materiales y Modelos de la Facultad de Ingeniería y Ciencias Aplicadas de la Universidad Central del Ecuador y corresponden a una réplica.

\begin{tabular}{|c|c|c|c|c|c|}
\hline $\begin{array}{c}\text { Orden al } \\
\text { Azar }\end{array}$ & $\begin{array}{l}\text { Orden } \\
\text { Estándar }\end{array}$ & $\begin{array}{c}\text { Factor 1: } \\
\text { AB (\%) }\end{array}$ & $\begin{array}{l}\text { Factor 2: } \\
\text { MFAF }\end{array}$ & $\begin{array}{c}\text { Factor 3: } \\
\text { MFAG }\end{array}$ & $\begin{array}{c}\text { Factor 4: } \\
\text { TC }\end{array}$ \\
\hline 1 & 4 & +1 & +1 & -1 & +1 \\
\hline 2 & 2 & +1 & -1 & -1 & +1 \\
\hline 3 & 11 & -1 & +1 & -1 & -1 \\
\hline 4 & 9 & -1 & -1 & -1 & -1 \\
\hline 5 & 7 & -1 & +1 & +1 & +1 \\
\hline 6 & 12 & +1 & +1 & -1 & -1 \\
\hline 7 & 16 & +1 & +1 & +1 & -1 \\
\hline 8 & 8 & +1 & +1 & +1 & +1 \\
\hline 9 & 3 & -1 & +1 & -1 & +1 \\
\hline 10 & 6 & +1 & -1 & +1 & +1 \\
\hline 11 & 5 & -1 & -1 & +1 & +1 \\
\hline 12 & 10 & +1 & -1 & -1 & -1 \\
\hline 13 & 1 & -1 & -1 & -1 & +1 \\
\hline 14 & 14 & +1 & -1 & +1 & -1 \\
\hline 15 & 15 & -1 & +1 & +1 & -1 \\
\hline 16 & 13 & -1 & -1 & +1 & -1 \\
\hline
\end{tabular}

\section{Diseño de Mezclas de Hormigón}

La dosificación empleada corresponde a la recomendada en la Guía de Diseño 7 (Ministerio de Desarrollo Urbano y Vivienda \& Secretaria de Gestión de Riesgos, 2016) para losas, vigas y columnas según se indica en la tabla 2. Seis muestras cilíndricas estándares de $15 \mathrm{~cm}$ x $30 \mathrm{~cm}$ fueron fabricadas de acuerdo a la normativa ASTM (2019a) para cada mezcla de hormigón para posteriormente ser ensayadas a cargas axiales de compresión a los 28 días de acuerdo a la normativa ASTM (2018). Para todas las mezclas se mantuvo constante la consistencia del hormigón a $7 \mathrm{~cm} \pm 1 \mathrm{~cm}$ en concordancia con la norma ASTM (2015). Cada mezcla fue realizada siguiendo un orden al azar, mismo que es diferente al orden estándar (ver Tabla 1), garantizando la independencia de cada experimento.

\begin{tabular}{|c|c|c|c|}
\hline $\begin{array}{l}\text { Agua a } \\
\text { (litros) }\end{array}$ & $\begin{array}{l}\text { Cemento } \\
(\mathrm{Kg})\end{array}$ & $\begin{array}{c}\text { Arena } \\
\left(\text { Parihuela }^{\mathrm{b}}\right)\end{array}$ & $\begin{array}{c}\text { Ripio } \\
\left(\text { Parihuela }^{\mathrm{b}}\right)\end{array}$ \\
\hline 25 & 50 & 2.0 & 2.5 \\
\hline
\end{tabular}

\section{Resultados y Discusión}

La tabla 3 contiene los resultados del diseño factorial $2^{4}$ con valores reales (no codificados) y la respuesta para cada experimento; es decir, la resistencia a la compresión $\left(f^{\prime} c\right)$ medida en mega pascales (MPa). El valor de $f^{\prime} c$ corresponde al promedio de la resistencia de seis muestras estándares cilíndricas ensayadas a esfuerzos de compresión axiales a la edad de 28 días. En la Norma Ecuatoriana de la Construcción para Estructuras de Hormigón Armado (Ministerio de Desarrollo Urbano y Vivienda \& Cámara de la Industria de la Construcción, 2015) se indica que el valor mínimo de la resistencia especificada del hormigón normal a ser utilizado para la fabricación de hormigón 
armado es de $21 \mathrm{MPa}$; razón por la cual, se tomará este valor como base de referencia para la dosificación establecida por la Guía de Diseño 7 (Ministerio de Desarrollo Urbano y Vivienda \& Secretaria de Gestión de Riesgos, 2016). Dicho de otra manera, se asumirá que $21 \mathrm{MPa}$ es la resistencia deseada y se calculará el Impacto (Tabla 3) de los módulos de finura del agregado fino y grueso, el \% de abrasión del agregado grueso y el tipo de cemento, como un porcentaje positivo o negativo dependiendo si dichos factores incrementan o reducen el valor de f'c según la ecuación (2).

$$
\text { Impacto }=\frac{f^{\prime} c_{\text {estimado }}-21}{21} * 100
$$

Tabla 3: Resultados del diseño factorial $\left(2^{4}\right)$ y el Impacto correspondiente en la resistencia a la compresión del hormigón $\left(f^{\prime} c\right)$

\begin{tabular}{cccccccc}
$\begin{array}{c}\text { Orden al } \\
\text { Azar }\end{array}$ & $\begin{array}{c}\text { Orden } \\
\text { Estándar }\end{array}$ & $\begin{array}{c}\text { AB } \\
(\%)\end{array}$ & MFAF & MFAG & TC & $\begin{array}{c}\text { f'c } \\
\text { MPa }\end{array}$ & $\begin{array}{c}\text { Impacto } \\
(\%)\end{array}$ \\
\hline 1 & 4 & 50.8 & 3.4 & 6.6 & GU & 19.93 & -5.1 \\
2 & 2 & 50.8 & 2.1 & 6.6 & GU & 20.02 & -4.7 \\
3 & 11 & 15.6 & 3.4 & 6.6 & IP & 23.60 & 12.4 \\
4 & 9 & 15.6 & 2.1 & 6.6 & IP & 19.00 & -9.5 \\
5 & 7 & 15.6 & 3.4 & 7.3 & GU & 25.49 & 21.4 \\
6 & 12 & 50.8 & 3.4 & 6.6 & IP & 20.58 & -2.0 \\
7 & 16 & 50.8 & 3.4 & 7.3 & IP & 21.05 & 0.2 \\
8 & 8 & 50.8 & 3.4 & 7.3 & GU & 22.49 & 7.1 \\
9 & 3 & 15.6 & 3.4 & 6.6 & GU & 26.97 & 28.4 \\
10 & 6 & 50.8 & 2.1 & 7.3 & GU & 23.79 & 13.3 \\
11 & 5 & 15.6 & 2.1 & 7.3 & GU & 23.07 & 9.9 \\
12 & 10 & 50.8 & 2.1 & 6.6 & IP & 18.12 & -13.7 \\
13 & 1 & 15.6 & 2.1 & 6.6 & GU & 20.00 & -4.8 \\
14 & 14 & 50.8 & 2.1 & 7.3 & IP & 20.07 & -4.4 \\
15 & 15 & 15.6 & 3.4 & 7.3 & IP & 24.00 & 14.3 \\
16 & 13 & 15.6 & 2.1 & 7.3 & IP & 22.83 & 8.7 \\
\hline
\end{tabular}

La tabla 4 contiene los resultados de los valores de los efectos y coeficientes calculados para cada factor y combinación de factores en unidades codificadas $(-1 \mathrm{o}+1)$. Esto se debe a que las unidades de cada factor son diferentes y si se trabajaría en unidades reales puede ocasionar distorsión en los resultados. El efecto calculado para el factor Abrasión (AB), por ejemplo, es igual a -2.364 lo que significa que cuando aumenta la abrasión disminuye el $f^{\prime} c$. En relación al valor de los coeficientes del modelo de regresión, estos corresponden a la mitad del efecto calculado, es decir, 1.182 en este caso para la abrasión. Todos los factores y combinación de factores que se indican son estadísticamente significativos ya que su valor $p$ es menor que 0.05 . Si el valor de $p$ fuera mayor que 0.05 significa que dicho término no es significativo y debe ser descartado del modelo ya que no se puede descartar la hipótesis nula $\left(\mathrm{Ho}: \beta_{i}=0\right)$. Dicho de otra manera, no hay suficiente evidencia para afirmar que el referido término tiene un valor diferente de cero.

Tabla 4: Efectos y Coeficientes del Modelo de Regresión en unidades codificadas $(-10+1)$.

\begin{tabular}{lcccc}
\multicolumn{1}{c}{ Término } & Efecto & Coeficiente & Valor $-\mathrm{t}$ & Valor $-\mathrm{p}$ \\
\hline Constante & & 21.938 & 84.12 & 0.000 \\
$\mathrm{AB}$ & -2.364 & -1.182 & -4.53 & 0.001 \\
MFAF & 2.151 & 1.076 & 4.12 & 0.003 \\
MFAG & 1.821 & 0.911 & 3.49 & 0.007 \\
TC & -1.564 & -0.782 & -3.00 & 0.015 \\
AB ${ }^{*}$ MFAF & -1.639 & -0.819 & -3.14 & 0.012 \\
MFAF ${ }^{*}$ MFAG & -1.334 & -0.667 & -2.56 & 0.031 \\
\hline
\end{tabular}

El modelo matemático final para estimar el valor de $f^{\prime} c$ en unidades reales (no codificadas) está representado por la ecuación (3). El coeficiente de determinación calculado $\left(R^{2}\right)$ es igual a 89.3\%, lo 
cual significa que es capaz de estimar valores de $f^{\prime} c$ de una manera adecuada, con una desviación estándar de 1.04 MPa.

$$
\begin{gathered}
f^{\prime} c_{\text {estimado }}=-61.0+0.1298 * A B+24.4100 * M F A F+10.6600 * M F A G-0.7820 * T C-0.0716 * A B * \\
M F A F-2.9300 * M F A F * M F A G
\end{gathered}
$$

El análisis de los residuales se lo debe realizar con el fin de comprobar si los residuales se encuentran normalmente distribuidos, si existe independencia entre experimentos (mezclas) y si las varianzas son iguales. Un valor residual es la diferencia entre el valor experimental y el estimado por el modelo matemático (ecuación (3)). Luego de realizar la prueba de normalidad Anderson - Darling (AD) para los residuales se tiene un valor estadístico $\mathrm{AD}=0.233$ con un valor de $p=0.756$ que es mayor que 0.05, lo que significa que los residuales siguen una distribución normal en razón de que no se puede descartar la hipótesis nula que señala que los residuales siguen una distribución normal. Realizar experimentos de manera aleatoria asegura que se mantenga la independencia y se la puede identificar cuando existe una tendencia marcada de valore positivos y negativos de los residuales, lo cual no ocurre en este caso. Luego de ejecutar la prueba de Bartlett para varianzas iguales se obtuvo un valor estadístico de 5.50 y un valor de $p=0.600$ que es mayor a 0.05 , lo que implica que todas las varianzas son iguales. De lo cual se desprende que el modelo matemático obtenido es adecuado.

Del referido modelo representado por la ecuación (3) y a través de la ecuación (2) se puede obtener el Impacto que tienen los agregados y tipo de cemento en la resistencia a la compresión $\left(f^{\prime} c\right)$ cuando se utilizan agregados particulares, con sus características propias. Además, se puede elaborar gráficos de líneas de contorno con el propósito de obtener o investigar zonas óptimas o de trabajo deseado; o a su vez, encontrar zonas con rango de $f^{\prime} c$ deseado manteniendo constantes dos de las cuatro variables. La figura 2 ilustra un ejemplo de lo indicado para un rango deseado de $f^{\prime} c$, donde MFAG y TC permanecen constantes. Los factores que pueden variar son el MFAF y la AB. Como se puede observar, en el caso de la figura 2.a), se mantienen constantes MFAG=6.7 y TC=IP y la zona en color blanco representa el espacio donde se puede obtener un hormigón de $20.5 M P a \leq f^{\prime} c \leq$ $21.5 \mathrm{MPa}$ para diferentes valores del MFAF y de AB. Es así que para un valor del MFAF=2.6 y $\mathrm{AB}=20 \%$, por ejemplo, le corresponde un $f^{\prime} \mathrm{C}=20.9 \mathrm{MPa}$. Igualmente, en la figura 2.b) donde cambia solamente el tipo de cemento ( $\mathrm{TC}=\mathrm{GU}$ ), se observa que dicha zona deseada se ubica en la parte inferior del gráfico, lo cual implica que el valor de $f^{\prime} c$ esperado, para las mismas variables (MFAG=6.7, MFAF=2.6 y AB=20\%) ahora aumenta a un valor de $f^{\prime} c=22.5 \mathrm{MPa}$. Un valor superior al que se espera. Es así como los constructores podrían detectar la influencia de las características de los materiales y tipo de cemento en el valor de $f^{\prime} c$ y de esta manera hacer uso de dichas curvas de contorno.

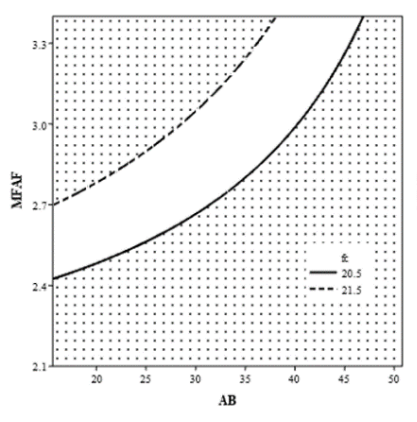

(a)

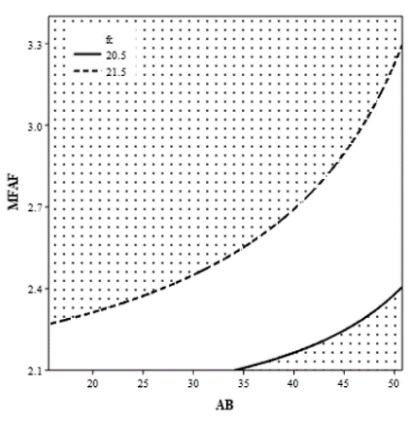

(b)

Figura 2: Zonas para 20.5MPa $\leq f^{\prime} \mathrm{c} \leq 21.5 \mathrm{MPa}$ para a) MFAG=6.7 y TC=IP, y b) MFAG=6.7 y TC=GU 


\section{Conclusiones}

Utilizando la teoría de DOE se puede analizar el impacto que tienen las propiedades de los agregados; módulos de finura de agregado fino y grueso, resistencia a la abrasión del agregado grueso y el tipo de cemento, en la resistencia a la compresión del hormigón $\left(f^{\prime} c\right)$ cuando se utiliza una misma dosificación al volumen. De los resultados contenidos en la tabla 3 se observa que el impacto es significativo afectando la resistencia a la compresión del hormigón $\left(f^{\prime} c\right)$ en un rango de $-13.7 \%$ a $28.4 \%$, lo cual implica que el personal profesional (ingenieros civiles y arquitectos) y no profesional (maestros mayores y albañiles) de la construcción deben estar al tanto de este particular ya que la calidad del hormigón que fabrican puede verse afectada considerablemente en la calidad de sus obras civiles o proyectos de construcción. El hormigón que se utiliza en la construcción de estructuras de hormigón armado debe ser el producto de un diseño de mezclas a fin de cumplir una especificación técnica emitida por el profesional estructural (ingeniero estructural), donde se consideren las propiedades de sus componentes y a partir de aquello llegar a establecer una dosificación al peso y luego la correspondiente dosificación al volumen.

El modelo matemático obtenido para estimar la resistencia a la compresión del hormigón representado por la ecuación (3) constituye una herramienta útil para determinar o analizar posibles zonas de interés con el fin de que no se vea afectada la calidad del hormigón deseado (Figura 2). El referido modelo matemático permitirá, además, al personal encargado de fabricar hormigón realizar una selección apropiada de los materiales a utilizarse, basado en criterios técnicos (resultados de ensayos o pruebas de laboratorio) y no solamente en la cercanía de los proveedores y costos de los mismos. Cabe indicar que las limitaciones del referido modelo se refieren a las características de los materiales pétreos utilizados; es decir, que la ecuación (3) es válida únicamente cuando las variables MFAF, MFAG y AB se encuentran dentro de los limites establecidos en la Sección 2.1. De manera similar, para el tipo de cemento GU y IP.

La metodología aplicada en este estudio puede ser extrapolada y utilizada para futuras investigaciones en diferentes locaciones geográficas que traten de determinar el impacto de otros factores sean estos las propiedades de los materiales componentes del hormigón o factores externos (humedad relativa) que actúan durante la fabricación de hormigón en el sitio de la obra. Todo esto siempre y cuando se utilice una misma dosificación; en caso que se desee estudiar proporciones de los componentes del hormigón a fin de encontrar mezclas optimas, se debe utilizar la teoría de diseño de experimentos para mezclas.

\section{Contribución de los autores}

En concordancia con la taxonomía establecida internacionalmente para la asignación de créditos a autores de artículos científicos (https://casrai.org/credit/). Los autores declaran sus contribuciones en la siguiente matriz:

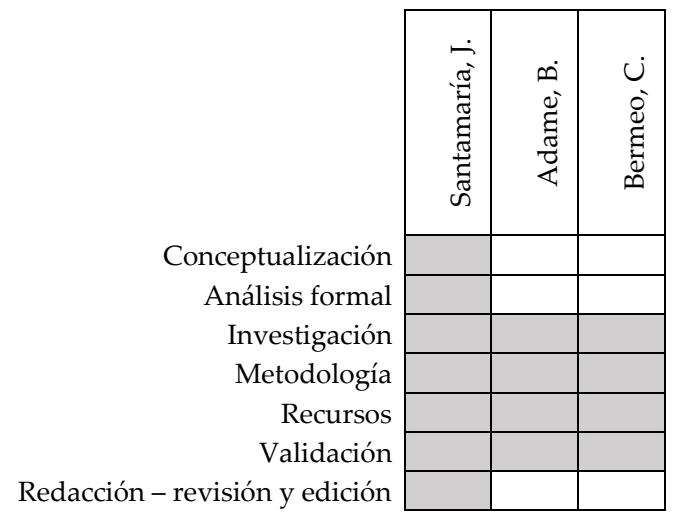




\section{Conflicto de Interés}

Los autores declaran que no existen conflictos de interés de ninguna naturaleza.

\section{Referencias}

ACI. (2009). Práctica estándar para seleccionar proporciones para concreto normal, pesado y masivo (ACI PRC-211.1-91). USA: American Concrete Institute.

ASTM. (2006). Método de prueba estándar para la resistencia a la degradación de agregado grueso de tamaño pequeño por abrasión e impacto en la máquina de Los Ángeles (ASTM C131 / C131M-14). West Conshohocken, PA, USA: ASTM International.

ASTM. (2015). Método de prueba estándar para asentamiento de concreto de cemento hidráulico (ASTM C143 / C143M-15aㅜ). West Conshohocken, PA, USA: ASTM International.

ASTM.(2018). Método de prueba estándar para resistencia a la compresión de muestras de concreto cilíndrico (ASTM C39 / C39M-18). West Conshohocken, PA, USA: ASTM International.

ASTM. (2019a). Práctica estándar para fabricar y curar muestras de ensayo de concreto en el campo (ASTM C31 / C31M-19a). West Conshohocken, PA, USA: ASTM International.

ASTM. (2019b). Método de prueba estándar para análisis por tamizado de agregados finos y gruesos (ASTM C136 / C136M-19). West Conshohocken, PA, USA: ASTM International.

ASTM. (2019c). Especificación estándar para cementos hidráulicos mezclados (ASTM C595 / C595M-19). West Conshohocken, PA, USA: ASTM International.

ASTM. (2020). Especificación de rendimiento estándar para cemento hidráulico (ASTM C1157 / C1157M$\left.20^{\underline{a}}\right)$. West Conshohocken, PA, USA: ASTM International.

Cornell, J. A. (2011). Experiments with mixtures: designs, models, and the analysis of mixture data (3rd ed.). New York, USA: John Wiley \& Sons.

De Larrard, F. (2011). Concrete mixture proportioning: a scientific approach. London and New York: Taylor \& Francis.

Carvajal, A. M. (14 de agosto de 2019). El 45\% de las casas informales de Quito tiene mayor riesgo sísmico. El Comercio. Recuperado de: https://www.elcomercio.com/actualidad/construccioninformal-sismos-vulnerabilidad-estudio.html

Ministerio de Desarrollo Urbano y Vivienda \& Secretaria de Gestión de Riesgos. (2016). Guía práctica de la construcción para no profesionales: Aplicando la norma ecuatoriana de la construcción NEC 2015. Recuperado de:

https://www.habitatyvivienda.gob.ec/wp-content/uploads/downloads/2016/10/GUIA-7trabajadores-no-profesionales1.pdf

Kosmatka, S. H., Kerkhoff, B., \& Panarese, W. C. (2011). Design and control of concrete mixtures (14 ${ }^{\text {th }}$ ed.). Skokie, IL, USA: Portland Cement Association.

Mehta, P. K., \& Monteiro, P. J. (2006). Concrete: microstructure, properties, and materials (3 ${ }^{\text {rd }}$ ed.). New York, USA: McGraw-Hill Education.

Montgomery, D. C. (2017). Design and analysis of experiments (9 $9^{\text {th }}$ ed.). River Street, Hoboken, NJ, USA: John Wiley \& Sons. 
Municipio de Quito. (2019). Ordenanza metropolitana No 004: Ordenanza metropolitana reformatoria del código municipal para el distrito metropolitano de quito, por la cual se incorpora la reglamentación sobre el reconocimiento y/o regularización de edificaciones existentes. Recuperado de: http://www7.quito.gob.ec/mdmq_ordenanzas/Ordenanzas/ORDENANZAS\%20MUNICIP ALES\%202019/ORD-MET-2019-004-

REGULARIZACION\%20EDIFICACIONES\%20EXISTENTES-INFORMALES-REFCODIGO\%20MUNICIPAL.pdf

Ministerio de Desarrollo Urbano y Vivienda \& Cámara de la Industria de la Construcción. (2015). Norma Ecuatoriana de la Construcción. NEC-SE-HM. Estructuras de Hormigón Armado. Recuperado de:

https://www.habitatyvivienda.gob.ec/wp-content/uploads/downloads/2015/02/NEC-SEHM-Hormig\%C3\%B3n-Armado.pdf

Holcim. (2020). Cemento Holcim Fuerte Tipo GU. Recuperado de:https://solucionesholcim.com/holcim-fuerte/

Unacem. (2020). Selvalegre - Decisiones que perduran. Recuperado de:https://unacem.com.ec/es/productos/selvalegre/ 